\title{
ÉTUDE COMPARÉE DE L'ACTION DES HORMONES HYPOPHYSAIRES ET STÉROÏDES SUR LA MATURATION IN VITRO DES OVOCYTES DE LA TRUITE ET DU CARASSIN (POISSONS TÉLÉOSTÉENS)
}

\author{
B. JALABERT, C. BRY, D. SZÖLLÖSI et A. FOSTIER \\ avec la collaboration technique de Marie-Claire Thtron et Anne-Marie Escafrre \\ Laboratoire de Physiologie des Poissons, I. N.R. A., \\ 78350 Jouy en Josas (France)
}

\begin{abstract}
RÉSUMÉ
La recherche de traitements gonadotropes ou stéroïdes susceptibles de déclencher ou d'inhiber in vitro la maturation ovocytaire chez la Truite et le Carassin, a conduit aux résultats suivants :

\section{Action des extraits gonadotropes hypophysaires sur les ovocytes entourés de tissu ovarien}

Chez le Carassin, l'extrait hypophysaire total de Carpe et l'hormone gonadotrope de Carpe $(c-\mathrm{HG}) n^{\prime}$ ont provoqué respectivement que $18 \mathrm{p}$. 100 et $26 \mathrm{p}$. roo de maturations pour les doses les plus élevées (tabl. 2), alors que chez la Truite l'extrait hypophysaire total homologue ou la préparation gonadotrope de Saumon induisent roo p. roo de maturations pour des doses bien plus faibles (JALABERT et al., I972). La métopirone (100 $\mu \mathrm{g} / \mathrm{ml})$ inhibe totalement l'action de l'extrait hypophysaire.
\end{abstract}

\section{Action des hormones stéroïdes}

Chez la Truite, la $20 \beta$-dihydroprogestérone est de loin le stéroïde le plus actif sur la maturation des ovocytes entourés de leurs enveloppes folliculaires (fig. I et 2). C'est en outre le seul stéroìde qui demeure actif sur les ovocytes dénudés après traitement enzymatique (tabl. 3).

Chez le Carassin, plusieurs stéroides ont une efficacité peu différente (cortéxolone, désoxycorticostérone, progestagènes). Cependant, leur action est presque complètement inhibée par la métopirone, à l'exception de la désoxycorticostérone (tabl. 4 et 5 ).

Ces résultats fournissent des présomptions en faveur de l'existence de médiateurs naturels de maturation ovocytaire, voisins respectivement de la désoxycorticostérone chez le Carassin et de la $20 \beta$-dihydroprogestérone chez la Truite.

Alors que chez la Truite ce médiateur peut être produit dans l'ovaire seul après stimulation gonadotrope, la participation d'un relai extra-ovarien (éventuellement interrénal) n'est pas à exclure chez le Carassin. 
Nous avons récemment mis en évidence que des extraits hypophysaires de Poissons et divers progestagènes sont susceptibles de déclencher in vitro la maturation des ovocytes de la Truite arc-en-ciel (Salmo gairdneri) prélevés et mis en incubation au stade de la " vésicule germinale périphérique " (JALABERT, BRETON, BRY, I972). La réponse aux extraits hypophysaires, plus tardive que la réponse aux progestagènes, exige en outre, au contraire des progestagènes, la présence de tissu ovarien enveloppant les follicules. Tout se passe comme si les progestagènes exogènes mimaient l'action d'un médiateur produit normalement par le tissu ovarien et actif sur la maturation ovocytaire.

Cette situation est très proche de celle des Amphibiens (voir revues de Schuerz, I969, I972 ; SMITH et ECKER, I970) chez lesquels 1'action d'un médiateur produit in vitro par le follicule lui-même (MASUI, I967), peut être mimée expérimentalement par divers progestagènes (SchUETz, I967 $a, \mathrm{I} 967 b, \mathbf{1 9 7 1}$; SuBTELNY et al., I968 ; SMith et ECKER, I97I). Par contre, elle diffère profondément du schéma établi chez un autre Poisson Téléostéen, Heteropneustes fossilis (Sundararaj et Goswam, I966 $a$ et $b$, I97I ; Goswami et Sundararaj, I97I $a$ et $b$; SundararaJ, ANAND et Donaldson, I972 ; SundararaJ, Goswami et Donaldson, I972), selon lequel les hormones gonadotropes mammaliennes o-LH, HCG, la gonadotropine de Saumon, pourtant actives in vivo sur la maturation ovocytaire et l'ovulation, sont inactives in vitro ou ne présentent qu'une faible action marginale; la stimulation gonadotrope serait en fait relayée par l'organe interrénal qui produirait de la désoxycorticostérone et de l'hydrocortisone agissant en synergie sur la maturation, dans un rapport de concentration déterminé, différent des taux de sécrétion normaux de cet organe sous un contrôle de type ACTH.

Devant les différences importantes constatées dans le contrôle du processus de maturation entre la Truite et le Poisson-chat Heteropneustes fossilis, nous avons cherché à étudier les conditions de maturation in vitro chez un Poisson appartenant à une autre famille : le Carassin, Carassius auratus (Cyprinidé), en les comparant à celles de la Truite.

\section{MATÉRIEL ETT MÉTHODES}

Les femelles utilisées pour les expériences ont été sacrifiées pendant la période de reproduction naturelle : Truite arc-en-ciel (Salmo gairdneri) en novembre-décembre; Carassin, en maijuin. Les ovaires n'ont été conservés que lorsque les ovocytes étaient au stade de la vésicule germinale périphérique qui apparaît après la fin de la vitellogenèse et avant le déclenchement de la maturation et de l'ovulation. Ce " stade sensible", en dehors duquel il n'a jamais été possible de provoquer in vitro une maturation typique comparable à l'état de maturation naturel, dure plusieurs jours chez la Truite (fig. 3), mais est extrêmement fugace chez le Carassin où il ne peut être observé que difficilement sous loupe binoculaire grâce à un éclairage intense par transparence et sur une faible proportion des ovocytes d'un même ovaire.

Les incubations ont été réalisées en solutions physiologiques respectant sensiblement l'équilibre des principaux cations du plasma de chaque espèce (tabl. I), à pH 7,2 à 7,5 sous une atmosphère de I $\mathrm{p}$. $100 \mathrm{CO}_{2}, 50 \mathrm{p}$. I $00 \mathrm{~N}_{2}, 49 \mathrm{p}$. I00 $\mathrm{O}_{2}$, à I $5^{\circ} \mathrm{C}$ pour la Truite et $25^{\circ} \mathrm{C}$ pour le Carassin. Les ovocytes dans leur follicule et éventuellement entourés de tissu ovarien ont été placés en incubation dans des coupelles de verre par lots de ro, I5, 20 ou 25 pour $2 \mathrm{ml}$ dans le cas de la Truite et de 50 à roo pour $\mathrm{I} \mathrm{ml}$ dans le cas du Carassin (diamètre moyen respectif des ovocytes : $4 \mathrm{~mm}$ et $\mathrm{I} \mathrm{mm}$ ).

Pour certains essais, les ovocytes de Truite ont été complètement dénudés de toutes leurs enveloppes folliculaires après incubation pendant $\mathrm{I} h \mathrm{~h}$ à $\mathrm{I} 5^{\circ} \mathrm{C}$ dans une solution mixte de trypsine $(5 \mathrm{mg} / \mathrm{ml}$ ) et de collagénase $(5 \mathrm{mg} / \mathrm{ml}$ ) (Sigma) dans le liquide physiologique d'incubation ; les 
TABLEAU I

Milieux d'incubation pour ovocytes de Truite et de Carassin

\begin{tabular}{|c|c|c|}
\hline & Truite & Carassin \\
\hline $\mathrm{NaCl}(g / 1) \ldots \ldots$ & 8,60 & 7,30 \\
\hline $\mathrm{KCl}(\mathrm{g} / \mathrm{l}) \ldots \ldots \ldots \ldots \ldots \ldots \ldots \ldots \ldots$ & 0,23 & 0,18 \\
\hline $\mathrm{MgSO}_{4}\left(7 \mathrm{H}_{2} \mathrm{O}\right)(\mathrm{g} / \mathrm{l}) \ldots \ldots$ & 0,07 & 0,07 \\
\hline $\mathrm{MgCl}_{2}\left(6 \mathrm{H}_{2} \mathrm{O}\right)(\mathrm{g} / \mathrm{l}) \ldots \ldots \ldots \ldots \ldots$ & 0,20 & 0,18 \\
\hline $\mathrm{CaCl}_{2}\left(2 \mathrm{H}_{2} \mathrm{O}\right)(\mathrm{g} / \mathrm{l}) \ldots \ldots \ldots \ldots \ldots \ldots$ & 0,50 & 0,35 \\
\hline $\mathrm{NaH}_{2} \mathrm{PO}_{4}(\mathrm{~g} / \mathrm{l}) \ldots \ldots \ldots \ldots \ldots \ldots \ldots \ldots \ldots \ldots \ldots \ldots \ldots$ & 0,12 & 0,08 \\
\hline $\mathrm{NaHCO}_{3}(\mathrm{~g} / \mathrm{l}) \ldots \ldots \ldots \ldots \ldots \ldots \ldots \ldots$ & 0,57 & 0,98 \\
\hline Glucose $(g / 1) \ldots \ldots \ldots \ldots \ldots \ldots \ldots \ldots$ & 1 & 1 \\
\hline Pression osmotique mesurée (m. osmomoles).. & 310 & 275 \\
\hline Température d'incubation $\left({ }^{\circ} \mathrm{C}\right) \ldots \ldots \ldots \ldots$ & 15 & 25 \\
\hline $\mathrm{pH}$ sous atmosphère $1 \% \mathrm{CO}_{2} \ldots \ldots \ldots \ldots$ & $7,3-7,5$ & $7,3-7,6$ \\
\hline
\end{tabular}

enveloppes folliculaires de nombreux ovocytes se fendent alors spontanément et sont déchirées et enlevées à l'aide de pinces fines. Les lambeaux d'épithélium folliculaire encore adhérents sont éliminés par un nouveau bain de 5 minutes dans la solution de trypsine-collagénase.

La préparation des extraits hypophysaires et la source des hormones gonadotropes ont été décrites par ailleurs (J ALABERT, BRETON et BRY, 1972). L'hormone gonadotrope de Carpe (c-HG) a été préparée par E. Burzawa-Gérard (I97I). Nous avons utilisé les hormones stéroïdes suivantes : prégnènolone (hydroxy- $3 \beta$-prégnène-5-one-20), progestérone (pregnène-4-dione-3, 20), I $7 \alpha$-hydroxyprogestérone (hydroxy-I $7 \alpha$-prégnène-4-dione, 3,20 ), $20 \alpha$-dihydroprogestérone (hydroxy-2o $\alpha$-prégnène-4-one-3), 2o $\beta$-dihydroprogestérone (hydroxy-2o $\beta$-prégnène-4-one-3) cortexolone (dihydroxy-I $7 \alpha, 2 \mathrm{I}$-prégnène-4-dione-3, 20), désoxycorticostérone (hydroxy-2I prégnène-4-dione 3,20 ), acétate de désoxycorticostérone, hydrocortisone (trihydroxy-I I $\beta$, I $7 \alpha$, 2 I-prégnène-4-dione-3, 20), cortisone (dihydroxy-I $7 \alpha, 2 \mathrm{I}$-prégnène-4-trione 3 , I I, 20), œestrone, I $7 \beta$-cstradiol, testostérone. La métopirone (méthyl-2 bis (3-pyridyl)-I, 2-propanone-I) nous a été fournie par la firme Ciba (SU ${ }_{4885}$ ).

\section{RÉSULTATS}

Nous avons déjà décrit les modifications macroscopiques liées à la maturation ovocytaire in vitro chez la Truite (JALABERT, BRETON, BRY, I972) (voir fig. 3, 4, 5).

Chez le Carassin dont les ovocytes en fin de vitellogenèse (diamètre voisin de I $\mathrm{mm}$ ) présentent un aspect blanchâtre ou jaunâtre, crayeux et opaque, la vésicule germinale est très difficile à distinguer. Aussi avons-nous conclu à la maturation ou à la non maturation par référence à l'aspect des œufs spontanément maturés et ovulés in vivo, extrêmement translucides (fig. 6). A $25^{\circ} \mathrm{C}$ la transformation, beaucoup plus rapide que chez la Truite, dure une vingtaine d'heures environ.

\section{Action des extraits hypophysaires et des hormones gonadotropes}

\section{Chez la Truite.}

Toutes les hormones et extraits hypophysaires de Poissons sont actifs, à des degrés divers, sur la maturation des ovocytes de Truite (JAIABERT, BRETON, BRY, I972). Signalons cependant que depuis nos précédentes observations nous avons pu vérifier une action positive de la $a-\mathrm{L} H$ pour des doses de l'ordre de $100 \mu \mathrm{g} / \mathrm{ml}$, alors que la dose de Io $\mu \mathrm{g} / \mathrm{ml}$ était inactive. 
2. Chez le Carassin (tab1. 2).

Les hormones hypophysaires ne sont jamais très actives dans notre système d'incubation : le meilleur résultat observé ( $26 \mathrm{p}$. Ioo de maturation) est obtenu grâce à 1'hormone gonadotrope de Carpe $(c-\mathrm{HG}$, Io $\mu \mathrm{g} / \mathrm{ml})$. L'extrait hypophysaire total

\section{TABLEAU 2}

Action d'extraits hypophysaires de Poissons et d'hormones gonadotropes sur la maturation in vitro des ovocytes de Carassin

(résultats obtenus sur une même femelle)

\begin{tabular}{|c|c|c|}
\hline Nature du traitement & Dose & $\begin{array}{l}\text { Pourcentage } \\
\text { de maturation } \\
\text { après } \\
24 \text { h d'incubation }\end{array}$ \\
\hline Extrait hypophysaire total de Carpe & $\begin{array}{r}25 \mu \mathrm{g} / \mathrm{ml} \\
50 \mu \mathrm{g} / \mathrm{ml} \\
100 \mu \mathrm{g} / \mathrm{ml} \\
200 \mu \mathrm{g} / \mathrm{ml}\end{array}$ & $\begin{array}{r}0 \\
3 \\
10 \\
18\end{array}$ \\
\hline $\begin{array}{l}\text { Extrait hypophysaire total de Carpe } \\
\text { en présence de métopirone } \\
(100 \mu \mathrm{g} / \mathrm{ml})\end{array}$ & $200 \mu \mathrm{g} / \mathrm{ml}$ & 0 \\
\hline Hormone de Carpe $(c-\mathbf{H G})$ & $10 \mu \mathrm{g} / \mathrm{ml}$ & 26 \\
\hline $\begin{array}{l}\text { Gonadotropine de Saumon } \\
\qquad(\mathrm{SG}-\mathrm{G} \text { 100) }\end{array}$ & $\begin{array}{r}12,5 \mu \mathrm{g} / \mathrm{ml} \\
25 \mu \mathrm{g} / \mathrm{ml} \\
50 \mu \mathrm{g} / \mathrm{ml} \\
100 \mu \mathrm{g} / \mathrm{ml} \\
200 \mu \mathrm{g} / \mathrm{ml}\end{array}$ & $\begin{array}{l}0 \\
1 \\
8 \\
8 \\
7\end{array}$ \\
\hline $\begin{array}{l}o-\mathrm{LH} \\
o-\mathrm{FSH} \\
\mathrm{HCG}\end{array}$ & 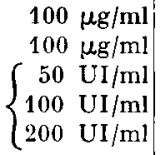 & $\begin{array}{l}0 \\
0 \\
2 \\
2 \\
2\end{array}$ \\
\hline
\end{tabular}

de Carpe à la dose de $200 \mu \mathrm{g} / \mathrm{ml}$ ne donne que I8 p. Ioo de maturation. La gonadotropine de Saumon (SG-G IOo) est encore moins efficace que celle de Carpe, avec au maximum $8 \mathrm{p}$. Ioo de maturations pour une dose de $50 \mu \mathrm{g} / \mathrm{ml}$.

$o$-LH et $o-F S H$ n'ont aucune action, même pour Ioo $\mu \mathrm{g} / \mathrm{ml}$. HCG induit au mieux 2 p. roo de maturation, ce qui contraste avec son activité pour provoquer maturation et ovulation in vivo.

\section{Action des hormones stéroïdes}

I. Chez la Truite (fig. I et 2, tabl. 3).

L'action des hormones stéroïdes, comme d'ailleurs celle des hormones hypophysaires, ne provoque pas forcément une réponse " tout ou rien ", en particulier pour les doses limites. Nous avons qualifié de " maturation vitelline " le phénomène 
net de fusion du vitellus et de migration périphérique des gouttelettes lipidiques, qui se traduit par une clarification du vitellus, la vésicule germinale étant encore présente, par opposition à la "maturation complète " lorsque la vésicule germinale a éclaté.

TABLEAU 3

Effet des hormones stéroïdes (I $\mu \mathrm{g} / \mathrm{ml}$ )

sur la maturation des ovocytes avec ou sans enveloppes folliculaires

\begin{tabular}{|c|c|c|c|c|}
\hline \multirow{3}{*}{ Nature du traitement } & \multicolumn{4}{|c|}{$\begin{array}{l}\text { Nombre de maturations completes }(+) \\
\text { ou incompletes }( \pm) \text { par lots de } 10\end{array}$} \\
\hline & \multicolumn{2}{|c|}{ Ovocytes avec follicule } & \multicolumn{2}{|c|}{ Ovocytes dénudés } \\
\hline & \pm & + & \pm & + \\
\hline $\begin{array}{c}\text { Témoin solvant } \\
\text { Désoxycorticostérone } \\
\text { Cortisone } \\
\text { Corticostérone } \\
\text { Cortexolone } \\
\text { Hydrocortisone } \\
\text { Progestérone } \\
17 \alpha \text {-hydroxyprogestérone } \\
20 \alpha \text {-dihydroprogestérone } \\
20 \beta \text {-dihydroprogestérone } \\
\text { Prégnénolone } \\
\text { CEstradiol } \\
\text { Testostérone }\end{array}$ & 4 & $\begin{array}{r}9 \\
10 \\
6 \\
10 \\
6\end{array}$ & 3 & 7 \\
\hline
\end{tabular}

Les figures I et 2 représentent respectivement les pourcentages de maturation vitelline et de maturation totale (vésicules germinales éclatées) observés sur des ovocytes non dénudés d'une même femelle, incubés par lots de 20 , en fonction de doses décroissantes d'hormones stéroïdes. Seuls sont mentionnés sur ces figures, les stéroïdes qui se sont révélés actifs. La désoxycorticostérone, active sur la maturation vitelline, n'a pas provoqué de maturations complètes et n'apparaît pas sur la figure 2 . Les comptages ont été effectués après 48 heures d'incubation à $I 5^{\circ} \mathrm{C}$, durée qui permet de discriminer au mieux les différences d'efficacité entre les stéroïdes. En fait, les pourcentages de maturation progressent dans le temps pour atteindre un maximum vers $72 \mathrm{~h}$, ce qui correspond à la limite de durée de l'incubation avant que les ovocytes ne commencent à dégénérer.

De ces observations il ressort qu'un progestagène particulier, la 2oß-dihydroprogestérone est très significativement plus efficace pour induire 1a maturation complète ou vitelline que le groupe des autres progestagènes entre lesquels les différences ne sont pas significatives.

Par ailleurs, lorsque des ovocytes dénudés par traitement enzymatique (tabl. 3) sont incubés en présence des mêmes stéroïdes, seule la $20 \beta$-dihydroprogestérone conserve son activité sur la maturation, avec cependant un retard de l'ordre de $\mathrm{I} 2 \mathrm{~h}$ sur les ovocytes non dénudés. 
- Progestérone

- $17 \alpha$-Hydroxy-progestérone

D $20 \propto$-Dihydro-progestérone
- $20 \beta$ - Dihydro-progesterone

$\triangle$ Prégnènolone

- Cortexolone

- Désoxy-corticostérone

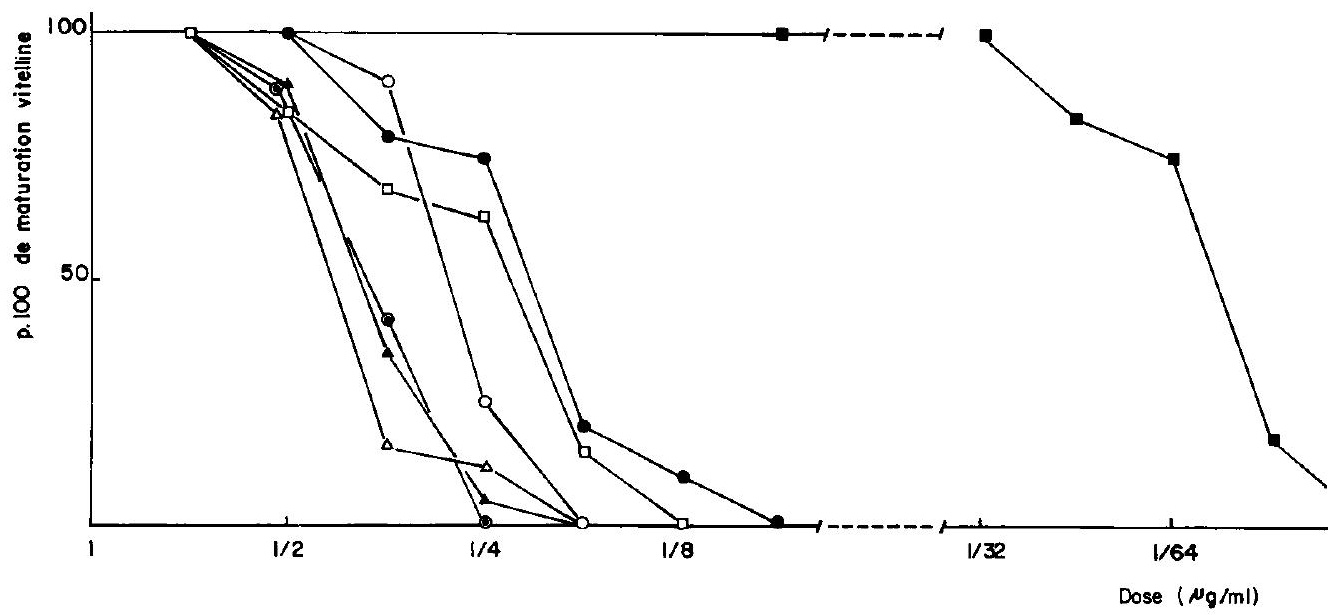

FIG. I. - Pourcentages de maturations vitellines (indépendamment de l'éclatement de la vésicule germinale)

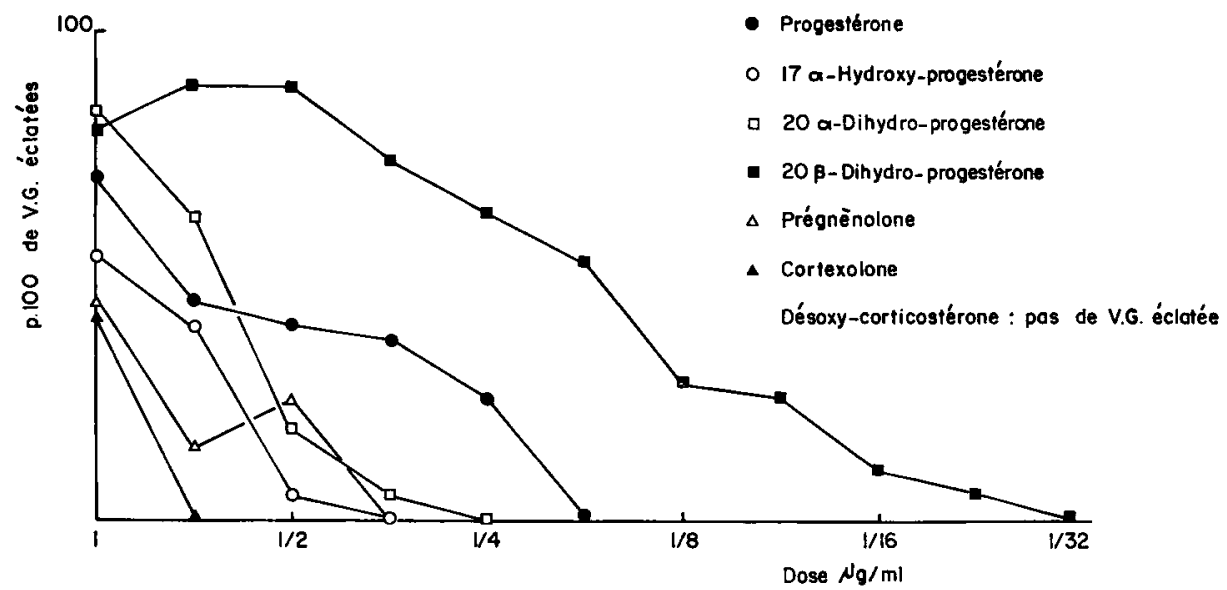

FIG. 2. - Pourcentages de maturations complètes (après disparition de la vésicule germinale)

Fig. I et 2. - Pourcentages de maturation observés dans des lots de 20 ovocytes d'une même femelle de Truite Arc-en-ciel, en fonction de doses décroissantes d'hormones stéroĩdes dans le milieu d'incubation (échelle logarithmique), après $48 h$ d'incubation à $15^{\circ} \mathrm{C}$. 
2. Chez le Carassin (tabl. 4 et 5 ).

Le tableau 4 montre le résultat des incubations réalisées avec les différents stéroïdes, en fonction de la dose.

Dans le groupe le plus actif on trouve les progestagènes, la désoxycorticostérone

\section{TABLEAU 4}

Action des hormones stéroïdes sur la maturation in vitro des ovocytes de Carassin

Pourcentages de maturation observés après $24 \mathrm{~h}$ d'incubation sur des ovocytes provenant de deux femelles différentes

\begin{tabular}{|c|c|c|c|c|c|c|c|c|c|}
\hline \multirow{2}{*}{ Nature du stéroïde } & \multirow{2}{*}{$\begin{array}{l}\text { Numéro } \\
\text { de la } \\
\text { donneuse }\end{array}$} & \multicolumn{8}{|c|}{ Dose $(\mu \mathrm{g} / \mathrm{ml})$} \\
\hline & & 4 & 2 & 1 & $1 / 2$ & $1 / 4$ & $1 / 8$ & $1 / 16$ & $1 / 32$ \\
\hline Cortexolone & 1 & 66 & 59 & 55 & 50 & 50 & 13 & 2 & 0 \\
\hline Désoxycorticostérone & 1 & 60 & 52 & 51 & 37 & 6 & 0 & 0 & 0 \\
\hline $\begin{array}{l}\text { Acétate de } \\
\text { désoxycorticostérone }\end{array}$ & $\begin{array}{l}1 \\
2\end{array}$ & $\begin{array}{l}49 \\
72\end{array}$ & $\begin{array}{l}43 \\
50\end{array}$ & $\begin{array}{l}49 \\
58\end{array}$ & $\begin{array}{l}45 \\
40\end{array}$ & $\begin{array}{l}50 \\
27\end{array}$ & $\begin{array}{l}21 \\
20\end{array}$ & $\begin{array}{l}4 \\
7\end{array}$ & $\begin{array}{l}0 \\
0\end{array}$ \\
\hline $20 \beta$-dihydroprogestérone & 2 & 43 & 37 & 30 & 10 & 8 & 3 & 0 & 0 \\
\hline 17 $\alpha$-hydroxyprogestérone & $\begin{array}{l}1 \\
2\end{array}$ & $\begin{array}{l}45 \\
46\end{array}$ & $\begin{array}{l}45 \\
42\end{array}$ & $\begin{array}{l}42 \\
40\end{array}$ & $\begin{array}{l}42 \\
39\end{array}$ & $\begin{array}{r}7 \\
19\end{array}$ & $\begin{array}{r}0 \\
14\end{array}$ & $\begin{array}{l}0 \\
2\end{array}$ & $\begin{array}{l}0 \\
0\end{array}$ \\
\hline $20 \alpha$-dihydroprogestérone & 2 & 31 & 27 & 27 & 10 & 5 & 0 & $\mathbf{0}$ & $\mathbf{0}$ \\
\hline Progestérone & $\begin{array}{l}1 \\
2\end{array}$ & $\begin{array}{l}45 \\
29\end{array}$ & $\begin{array}{l}36 \\
18\end{array}$ & $\begin{array}{l}37 \\
20\end{array}$ & $\begin{array}{r}8 \\
16\end{array}$ & $\begin{array}{l}4 \\
3\end{array}$ & $\begin{array}{l}0 \\
2\end{array}$ & $\begin{array}{l}0 \\
0\end{array}$ & $\begin{array}{l}0 \\
0\end{array}$ \\
\hline Cortisone & 1 & 30 & 7 & 2 & 0 & 0 & 0 & 0 & 0 \\
\hline Hydrocortisone & 1 & 20 & 2 & 0 & 0 & 0 & 0 & 0 & 0 \\
\hline Corticostérone & 1 & 7 & 0 & 2 & 0 & 0 & 0 & 0 & 0 \\
\hline Estrone & 2 & 0 & & & & & & & \\
\hline $17 \beta$-Estradiol & 2 & 0 & & & & & & & \\
\hline
\end{tabular}

et la cortexolone. Les différences entre les effets de la cortexolone et de la désoxycorticostérone, de la désoxycorticostérone et de la progestérone, ne sont pas significatives (test de $\chi^{2}$ corrigé) ; seule la différence entre cortexolone et progestérone est significative. Il n'est donc pas possible de conclure au rôle privilégié d'un stéroĩde particulier dans cette expérience.

Par contre, lorsque les incubations sont réalisées en présence de métopirone $(200 \mu \mathrm{g} / \mathrm{ml}$ ) qui inhibe probablement de nombreuses conversions entre stéroïdes à cette dose élevée, seule la désoxycorticostérone conserve $5^{\circ} \mathrm{p}$. Ioo de son activité, alors que l'action des autres stéroïdes est pratiquemment totalement inhibée. 
TABLEAU 5

Effet de la métopirone sur la réponse des ovocytes de Carassin aux hormones stéroides actives sur la maturation

\begin{tabular}{|c|c|c|}
\hline \multirow[b]{2}{*}{ Nature du stéroïde } & \multicolumn{2}{|c|}{ Pourcentage de maturation } \\
\hline & $\begin{array}{l}\text { Avec stéroïde seul } \\
\qquad(4 \mu \mathrm{g} / \mathrm{ml})\end{array}$ & $\begin{array}{l}\text { Avec stéroïde }(4 \mu \mathrm{g} / \mathrm{ml}) \\
\text { en présence de } \\
\text { métopirone }(200 \mu \mathrm{g} / \mathrm{ml})\end{array}$ \\
\hline Cortexolone .......... & 66 & 2 \\
\hline Désoxycorticostérone .... & 60 & 30 \\
\hline $17 \alpha$-hydroxyprogestérone. & 45 & 2 \\
\hline Progestérone ........ & 45 & 2 \\
\hline Cortisone............ & 30 & 0 \\
\hline Hydrocortisone ........ & 20 & 0 \\
\hline Corticostérone $\ldots \ldots \ldots$ & 7 & 3 \\
\hline
\end{tabular}

\section{DISCUSSION}

Nos résultats ne concernent que le processus de maturation ovocytaire et non celui de l'ovulation (émission des ovocytes maturés hors du follicule) qui en est disjoint dans les conditions expérimentales rapportées ici, alors que ces deux événements se succèdent nécessairement in vivo, sous contrôle gonadotrope hypophysaire (voir revues de PICKFORD et ATZ, I957 ; BALL, I960; HOAR, I969; et les travaux de Yamazakr, I965, chez le Poisson rouge et de SundaraRaj et Goswami, I966, chez le Poisson-chat Heteropneustes fossilis). Cependant, l'ovulation consécutive à la maturation in vitro a été obtenue chez Oryzias latipes par HIROSE (I97I et I972) et HIROSE et DONALDSON (I972) après stimulation par des hormones gonadotropes ou stéroïdes, et chez Heteropneustes fossilis par Goswami et SundararaJ (I97I) après stimulation par des hormones stéroïdes. L'absence d'ovulation in vitro observée chez la Truite et le Carassin après traitement gonadotrope ou stéroïde peut tenir à la fois à la technique d'incubation ou au choix des espèces ; la durée de survie des tissus en incubation et particulièrement du follicule est limitée ce qui peut exclure toute observation d'une réponse différée; mais la non-réponse observée peut aussi refléter l'absence, dans le système d'incubation, d'un relai produisant le ou les facteurs d'ovulation. Remarquons que la maturation ovocytaire a pu aussi être dissociée de l'ovulation in vitro chez les Amphibiens où ces processus se succèdent pourtant très rapidement (SubTel, Ny, SMrth et Ecker, I968; Alonso-BEdate et al., I97I).

En ce qui concerne le mode d'action de l'hormone gonadotrope sur la maturation ovocytaire, nous avons constaté que la maturation in vitro chez le Carassin n'est jamais complète ( $26 \mathrm{p}$. Ioo au plus pour Io $\mu \mathrm{g} / \mathrm{ml}$ de $c-\mathrm{HG}$ ) au contraire de la Truite (IOo p. IOo avec moins de I $\mu \mathrm{g} / \mathrm{ml}$ d'hormone de Saumon SG-G IOo), et bien que les ovocytes soient aussi entourés de tissu ovarien. Il serait possible que l'état des ovocytes incubés soit hétérogène mais $60 \mathrm{p}$. roo d'entre eux sont capables de répondre 
à l'action de la désoxycorticostérone. On peut donc penser que le Carassin est dans une situation intermédiaire entre le Poisson-chat et la Truite, et faire l'hypothèse que l'action gonadotrope est partiellement relayée par l'organe interrénal fournissant soit un précurseur proche du médiateur actif, soit le médiateur lui-même. Cette hypothèse serait d'autant plus plausible que c'est un corticostéroïde, la désoxycorticostérone qui mime le plus efficacement l'action du médiateur présumé, même en présence de métopirone; la métopirone est un inhibiteur de compétition, spécifique de la II $\beta$-hydroxylation à faible dose chez les Mammifères (CHART et SHEPPARD, I959; WILLIAMSON et O'DONNELL, I967), mais qui pourrait inhiber d'autres conversions entre corticostéroìdes aux doses élevées que nous avons employées. La perte d'efficacité relative de la désoxycorticostérone en présence de métopirone ( $30 \mathrm{p}$. Ioo de maturations au lieu de $60 \mathrm{p}$. Ioo) pourrait d'ailleurs s'expliquer par une compétition au niveau même des sites récepteurs.

Cependant, si le rôle éventuel de l'interrénal est possible il peut être totalement remis en question chez le Carassin, comme chez Heteropneustes fossilis : en effet, le pourcentage relativement faible de maturation in vitro chez le Carassin en réponse à une action gonadotrope, tout comme la très faible réponse observée chez Heteropneustes fossilis (Goswami et SundararaJ, 197 $\mathrm{x}$ ) peuvent seulement tenir à une insuffisance physiologique de l'ovaire en incubation ; le fait que la métopirone bloque totalement la réponse de maturation in vitro aux extraits hypophysaires chez le Carassin suggère que chez les Poissons son action inhibitrice pourrait ne pas être spécifique de l'organe interrénal et de la corticostéroïdogenèse, comme l'avaient supposé SundararaJ et Goswami (Ig66 $b$ ).

Chez la Truite, en tout cas, il ne paraît pas nécessaire d'impliquer l'organe interrénal directement dans le mécanisme de contrôle de la maturation, tout comme chez Oryzias latipes (HIROSE, I97I, I972) et chez les Amphibiens. En outre, le problème de savoir si l'hormone gonadotrope est seulement active par l'intermédiaire du médiateur folliculaire ou ovarien ou si elle a aussi une action propre sur l'ovocyte ne se pose pas comme chez les Amphibiens, où quelques cellules folliculaires seulement suffiraient à relayer 1'action gonadotrope (MASUI, 1967); en effet, l'action gonadotrope pour être efficace in vitro chez la Truite exige, outre la présence de follicules, celle de tissu ovarien qui sert de relai sans pour autant exclure une contribution partielle des enveloppes folliculaires.

Chez les Amphibiens, de nombreux faits expérimentaux in vivo et in vitro (revus par SCHUETZ, I969; SMITH et ECKER, I970) ont apporté la quasi-certitude que le médiateur de maturation est un stéroïde, vraisemblablement un progestagène. Son mode d'action prête cependant à controverses, soit qu'il agisse au niveau de la membrane ovocytaire en induisant la production d'un second messager hormonal dans le cytoplasme comme le suggèrent SMITH et ECKER (I970, I97I) après des essais infructueux d'injections de progestérone dans les ovocytes de Rana pipiens, soit qu'il pénètre effectivement dans le cytoplasme ovocytaire où il pourrait se fixer à une fraction protéique réceptrice (HORTON, I969) sachant par ailleurs que chez Xenopus laevis, la progestérone injectée dans l'ovocyte déclenche la maturation (SCHORDFRETSLATKINE, I972).

Chez les Poissons, nous commençons aussi à disposer d'un faisceau de présomptions : in vivo, depuis les premières observations de KIRSHENBLATT (1952, I959) sur l'action maturante de la méthyltestostérone et de la progestérone chez Misgurnus 
fossilis, signalons 1'action des corticostéroïdes chez Heteropneustes fossilis (RAMASwaMI, I962) et celle de la progestérone chez Carassius auratus (YAMAZAKI, I965) ; in vitro, la maturation ovocytaire a été induite par la cortisone chez Mirgunus fossilis (KIRSHENBLATT, I959), par la progestérone chez 1'Esturgeon, Acipenser stellatus (DETTLAFF et Skoblina, I969), par les corticostéroïdes et particulièrement un rapport synergique de doses entre la désoxycorticostérone et l'hydrocortisone chez Heteropneustes fossilis (Goswami et Sundararaj, I97I ; Sundararaj et Goswami, r97I), enfin par la progestérone et surtout l'hydrocortisone chez Oryzias latipes (HIROSE, I972).

Nos propres résultats chez le Carassin font jouer un rôle privilégié à la désoxycorticostérone, le seul des stéroïdes essayés dont 1'action n'est pas complètement inhibée par la métopirone, ce qui pourrait signifier que le médiateur naturel est identique ou très voisin. Chez la Truite, le rôle privilégié est joué par la 20ß-dihydroprogestérone qui est bien plus active sur la maturation vitelline que n'importe quel autre progestagène (fig. I et 2) et qui est en outre le seul stéroïde restant actif sur les ovocytes dénudés, sans enveloppes folliculaires (tabl. 2). Le léger retard observé (une dizaine d'heures dans la maturation par rapport aux ovocytes non dénudés peut tenir à une altération partielle de la membrane cytoplasmique ovocytaire lors du traitement enzymatique, ou bien au fait que l'absence du follicule empêche une métabolisation de la $20 \beta-$ dihydroprogestérone fournissant un produit plus actif. IDLER, FAGERLUND et RoNALD (I960) ont mis en évidence l'existence dans le plasma du Saumon, après la fraie, d'un stéroĩde particulier, la $20 \beta$-dihydro-I $7 \alpha$-hydroxyprogestérone, identifié pour la première fois in vivo. Il est à remarquer (tabl. 6, d'après ScHMIDT et IDLER,

\section{TABI,EAU 6}

Stéroïdes dans le plasma de Saumon (Onchorynchus nerka) d'après Schmidt et IdLER (I962) ( $\mu \mathrm{g} / \mathrm{I}$ oo ml)

\begin{tabular}{|c|c|c|c|c|}
\hline $\begin{array}{l}\text { Echantillon } \\
\text { et sexe }\end{array}$ & État de maturité sexuelle & 11 cétotestostérone & $\begin{array}{c}20 \beta \text {-dihydro- } \\
17 \alpha \text {-hydroxy- } \\
\text { progestérone }\end{array}$ & $\begin{array}{l}17 \alpha \text {-hydroxy- } \\
\text { progestérone }\end{array}$ \\
\hline 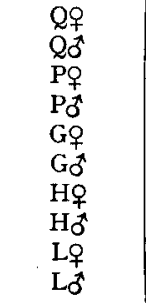 & $\begin{array}{c}\text { Juste avant début migration } \\
\text { Début migration } \\
85 \% \text { du voyage de migration } \\
\text { Matures. Avant la fraie } \\
\text { Après la fraie }\end{array}$ & $\begin{array}{r}2,5 \\
7,9 \\
7,1 \\
4,8 \\
0,6 \\
7,2 \\
8,7 \\
17,1 \\
0 \\
12,1\end{array}$ & $\begin{array}{r}0 \\
0 \\
0 \\
0 \\
0 \\
0 \\
23,9 \\
9,5 \\
13,4 \\
3,3\end{array}$ & $\begin{array}{l}0 \\
0 \\
0,6 \\
1,9 \\
3,8 \\
0 \\
20,2 \\
3,3 \\
13,6 \\
1,8\end{array}$ \\
\hline
\end{tabular}

Ig62) que ce stéroïde non identifiable chez les adultes en migration, atteint des taux importants, chez la femelle tout particulièrement, juste avant et juste après la fraie. Ces taux sont compatibles avec les doses de $20 \beta$-dihydroprogestérone utilisées effica- 
cement in vitro pour obtenir la maturation ovocytaire. Le véritable médiateur de la maturation ovocytaire chez les Salmonidés pourrait donc être très proche de ces deux stéroïdes.

\section{REMERCIEMENTS}

Nous remercions particulièrement B. Bonicel, M. BESNard et G. DAURAT pour les soins prodigués à nos élevages de Poissons sous la direction technique de M. CARPEnTIER, sans lesquels ce travail n'aurait pu être réalisé.

\section{SUMMARY}

COMPARATIVE STUDY OF PITUITARY

AND STEROID HORMONE ACTION ON IN VITRO MATURATION OF TROUT AND GOLDFISH (TEI,EOST FISH) OOCYTES

The search for gonadotrophic or steroid treatments capable of inducing or inhibiting in vitro oocyte maturation in the trout and goldfish, leads to the following points :

\section{Action of pituitary gonadotrophin extracts on oocytes surrounded by ovarian tissue}

In the goldfish, the highest doses (table 2) of total carp pituitary extract and carp gonadotrophic hormone ( $c-H G$ ) only induce I 8 p. I0o and 26 p. Ioo maturation, respectively, while in trout the homologous total trout pituitary extract or salmon gonadotrophic preparation induces Ioo p. Ioo maturation when very low doses are used (JALABERT et al., I972). Metopirone (IOo $\mu \mathrm{g} / \mathrm{ml})$ completely inhibits pituitary extract action.

\section{Action of steroid hormones}

In the trout, 2oß-dihydroprogesterone has, by far, the strongest steroid action on oocytes surrounded by their follicular membranes (fig. I and 2). Moreover, it is the only steroid which still acts on naked oocytes after enzymatic treatment (table 3 ).

In the goldfish, several steroids have almost the same effect (cortexolone, desoxycorticosterone, progestagens). However, their action is almost entirely inhibited by metopirone, with the exception of desoxycorticosterone (tables 4 and 5 ).

These results indicate that natural mediators of oocyte maturation may exist, which are similar, respectively, to desoxycorticosterone in the goldfish and $20 \beta$-dihydroprogesterone in the trout.

While the mediator in the trout may be produced in the ovary only, after gonadotrophic stimulation, the participation of an extra-ovarian relay (probably interrenal) should not be excluded in the goldfish.

\section{RÉFÉRENCES BIBL,IOGRAPHIQUES}

Alonso-Bedate M., Fraile A., Lopez-Gordo J. L., Calle C., I971. Action of progesterone on the maturation and ovulation of Discoglossus pictus oocytes (Amphibia anouro) : results obtained in vivo and in vitro. Acta Embryol. exp., I971, 177-186.

Ball J. N., 1960. Reproduction in female bony fishes. Symp. Zool. Soc. London, 1, 105-135.

Burzawa-Gerard E., I97I. Purification d'une hormone gonadotrope hypophysaire de Poisson téléostéen, la Carpe (Cyprinus carpio). Biochimie, 53, 545-552. 
Chart J. J, Sheppard H., I959. Pharmacology and biochemistry of some amphemone analogues and other adrenal inhibitors. J. Med. Pharm. Chem., 1, 407-44I.

Dettlaff T. A., Skoblina M. N., rg69. The role of germinal vesicle in the process of oocyte maturation in Anura and Acipenseridae. Ann. Embr. Morph., Suppl., 1, I33-I5I.

Goswami S. V., SUndararaj B. I., I97I. Temporal effects of ovine luteinizing hormone and desoxycorticosterone acetate on maturation and ovulation of oocytes of the catfish, Heteropneustes fossilis (BLOCH). An in vivo and in vitro study. J. exp. Zool., 178, 457-466.

Goswami S. V., Sundararaj, B. I., I97I. In vitro maturation and ovulation of oocytes of the catfish Heteropneustes fossilis (BLOCH). Effects of mammalian hypophyseal hormones, catfish pituitary homogenate, steroid precursors and metabolites, and gonadal and adrenocortical steroids. J. exp. Zool., $178,467-478$.

Hirose K., I97I. Biological study on ovulation in vitro of fish. I. Effects of pituitary and chorionic gonadotropins on ovulation in vitro of Medaka, Oryzias latipes. Bull. Japan Soc. Scient. Fish., 3, $585 \cdot 59 \mathrm{I}$.

Hirose K., r972 Biological study on ovulation in vitro of fish. IV. Induction of in vitro ovulation in Oryzias latipes oocyte using steroids. Bull. Japan Soc. Scient. Fish., 38, 457-463.

Hirose K., Donaldson E. M., I972. Biological study on ovulation in vitro of fish. III. The induction of in vitro ovulation of Oryzias latipes oocytes using salmon pituitary gonadotropin. Bull. Japan. Soc. Scient. Fish., 38, 97-100.

Hoar W. S., I969. Reproduction. In : Fish Physiology. W. S. Hoar et D. J. Randall Ed., Acad. Press, New York, 3, I-59.

Horton K., r969. Hormonal interactions in maturing eggs of Xenopus laevis. Am. Zool., 9, 6or602 (Abstr.).

IdLer D. R., FAgerlund U. H. M., Ronald A. P., I96o. Isolation of pregn-4-ene-I $7 \alpha, 20 \beta$-diol3-one from the plasma of Pacific Salmon. Biochem. Biophys. Res. Comm., 2, 133-137.

JALABERT B., BReton B., BRY C., I972. Maturation et ovulation in vitro des ovocytes de la Truite arc-en-ciel Salmo Gairdneri. C. R. Acad. Sci., Paris, 275, r139-1142.

Kirshenblatt I. D., I952. The action of steroid hormones in female vy'un (russe). Dokl. Akad. Nauk., U.S.S.R., 88, 629-632.

Kirshenblatt I. D., r959. Effect of cortisone on ovaries of the Loach (russe ; résumé anglais). Bjull. eskp. Biol. Med, 47, 108-1 22.

MASUI Y., I967. Relative roles of the pituitary, follicle cells, and progesterone in the induction of oocyte maturation in Rana pipiens. J. Exp. Zool., 166, 365-375.

Pickford G. E., ATz J. W., 1957. The physiology of the pituitary gland of fishes. Acad. Press., New York.

Ramaswami L. S, 1962. Endocrinology of reproduction in fish and frog. Gen. Comp. Endocrinol.. suppl., 1, 286-299.

Schmidt P. J., IDLER D. R., 1962. Steroid hormones in the plasma of Salmon at various states of maturation. Gen. Comp. Endocrinol., 2, 204-2r4.

Schorderet-Slatkine S., 1972. Action of progesterone and related steroids on oocyte maturation in Xenopus laevis. An in vitro study. Cell. Different., 1, I79-189.

Schuetz A. W., I967a. Effects of steroids on germinal vesicle of oocytes of the frog (Rana pipiens) in vitro. Proc. Soc. exp. Biol. Med., 124, 1307-1310.

Schuetz A. W., I967 b. Action of hormones on germinal vesicle breakdown in frog (Rana pipiens) oocytes. J. exp. Zool., 166, 347-354.

Schuetz A. W., 1969. Oogenesis : Processes and their regulation. Adv. Reprod. Physiol., 4, 99-I48.

SchUETZ A. W., I97x. In vitro induction of ovulation and oocyte maturation in Rana pipiens ovarian follicles : effects of steroidal and non steroidal hormones. J. exp. Zool., 178, 377-386.

Schuetz A. W., I972. Hormones and follicular functions. In Oogenesis. J. D. Biggers et A. W. Schuetz, Univ. Park. Press, Baltimore et Butterworths, Londres, 1972, 479-5 II.

Smith L. D., Ecker R. E., I969. Role of the oocyte nucleus in physiological maturation in Rana pipiens. Develop. Biol., 28r-309.

Smith L. D., Ecker R. E., I97o. Regulatory processes in the maturation and early cleavage of amphibian eggs. Curr. Top. Develop. Biol., 5, I-38.

Smity L. D., Ecker R. E., I97I. The interaction of steroids with Rana pipiens oocytes in the induction of maturation. Develop. Biol., 25, 232-247.

Subtelny S., Smith L. D., Ecker R. E., I968. Maturation of ovarian frog eggs without ovulation, J. exp. Zool., 168, 39-47.

SundararaJ B. I., Goswami S. V., I966 a. Effects of mammalian hypophysial hormones, placental gonadotrophins, gonadal hormones and adrenal corticosteroids in ovulation and spawning in hypophysectomized catfish Heteropneustes fossilis (BLOCH.). J. exp. Biol., 161, 287-296.

SundararaJ B. I., Goswami S. V., I 966 b. Effect of metopiron (SU 4885) on luteinizing hormone and corticosteroid-induced ovulation and spawning in hypophysectomized catfish Heteropneustes fossi lis (вLOch.). J. exp. Zool., 163, 49-54.

Sundararaj B. I., Goswami S. V,, I97I. Effects of desoxycorticosterone and hydrocortisone singly 
and in various combinations on in vitro maturation of oocytes of the catfish Heteropneustes fossilis (вLOCH.). Gen. Comp. Endocr., 17, 570-573.

Sundararaj B. I., Anand T. C., Donaldson E. M., I972. Effects of partially purified Salmon pituitary gonadotrophin on ovarian maintenance, ovulation and vitellogenesis in the hypophysectomized catfish Heteropneustes fossilis (вLOCH). Gen. Comp. Endocr., 18, 102-II4.

Sundarara J B. I., Goswami S. V., Donaldson E. M., 1972. Effect of salmon gonadotropin on in vitro maturation of oocytes of a catfish Heteropneustes fossilis, J. Fish. Res. Bd. Can., 29, $435-437$.

Williamson D. G., O'Donnell V. J., I967. Mechanism of metopirone inhibition of a soluble adrenal steroid I $\beta$-hydroxylase. Can. J. Biochem., 45, I53-163.

Yamamoto K., Yamazaki F., 1967. Hormonal control of ovulation and spermiation in goldfish. Gunma Symp. Endocrinol., 4, I3I-145.

YAMAZAKI F., I965. Endocrinological studies on the reproduction of the female goldfish Carassius auratus (L.) with special reference to the function of the pituitary gland. Mem. Fac. Fish. Hokkaido Univ., 13, 1-65. 


\section{PLANCHE I}

\section{FIG. 3}

Ovocytes de Truite arc-en-ciel au stade de la " vésicule germinale périphérique " (v.g.) enveloppés de leurs couches folliculaires, elles-mêmes entourées d'un fin réseau de capillaires sanguins (c.s.). Les ovocytes sont placés en incubation à ce stade. Les lots témoins conservent ce même aspect après 72 heures d'incubation à $I 5^{\circ} \mathrm{C}$.

\section{FIG. 4}

Ovocytes de Truite arc-en-ciel ayant subi la maturation complète in vitro, après 48 heures d'incubation sous 1'action de la $20 \beta$-dihydroprogestérone. La présence des enveloppes folliculaires est visualisée par les capillaires sanguins (c.s.). La vésicule germinale a disparu. Des gouttelettes lipidiques (g.1.) se sont condensées en périphérie, et l'ensemble du vitellus a pris un aspect translucide.

\section{FIG. 5}

Ovocytes de Truite arc-en-ciel ayant subi spontanément la maturation et l'ovulation in vivo. Noter l'absence des enveloppes folliculaires, et comparer à la figure 4 .

\section{FIG. 6}

Ovocytes de Carassin, dans leur follicule, avant et après maturation. Avant maturation (en haut) les ovocytes sont blanchâtres et très opaques. Ils deviennent brun jaunâtre et translucides après maturation (en bas). 
PLANCHE I
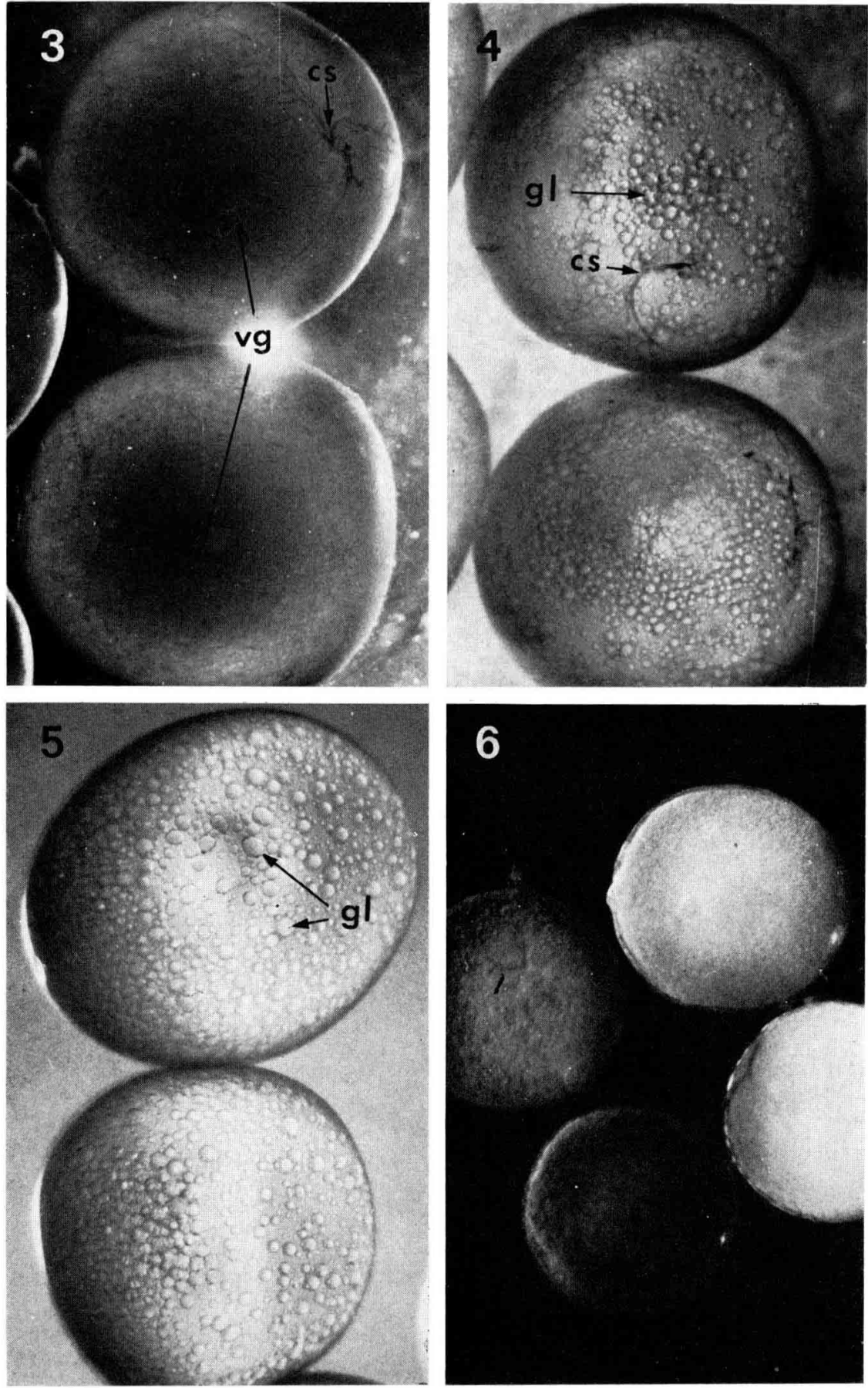

B. JALABERT, C. BRY, D. SZÖLLÖSI et A. FOSTIER 